\title{
NUMBERS OF REPRESENTATIONS OF INTEGERS IN A CERTAIN TRIAD OF TERNARY QUADRATIC FORMS*
}

BY

E. T. BELL

\section{INTRODUCTION AND SUMMARIES}

1. A powerful method concerning numbers of representations of integers in positive definite quadratic or bilinear forms is illustrated by a complete discussion for the three forms

$$
x y+y z+z x, x y+y z+2 z x, x y+2 y z+2 z x .
$$

The results for these forms are intrinsically interesting. It is a feature of the method that, in general, at least two forms are handled simultaneously. Complete results are attainable for all of the forms in a given set or for none. We are interested here only in obtaining final results involving nothing more abstruse than the binary quadratic class number. For the possibilities sketched in $\$ 7$ the class number is insufficient.

2. Without further remark, $a, b, c$ denote constant integers $>0$, and, unless otherwise stated, $x, y, z$ denote integers ranging $\geqq 0 ; n$ is an arbitrary (odd or even) constant integer $>0 ; m$ is an arbitrary odd constant integer $>0 ; \alpha$ is an arbitrary constant integer $\geqq 0$, so that $2^{\alpha} m$ is an arbitrary constant integer $>0$. If restrictions be imposed upon $m$, as for example $m \equiv 3$ $\bmod 8$, they will be indicated. Similarly for $x, y, z$.

The number of matrices $(x, y, z)$ such that $n=a x y+b y z+c z x$ is called, as usual, the number of representations of $n$ in the form $a x y+b y z+c z x$. This number will be denoted by $N_{0}[n=a x y+b y x+c z x]$. The number of solutions $(x, y, z)$ for which $x>0, y>0, z>0$, will be denoted by $N_{1}[n=$ $a x y+b y z+c z x]$. Let $\zeta(x) \equiv 0$, or the number of divisors of $x$, according as $x$ is not, or is, an integer $>0$. Then, evidently,

$$
\begin{aligned}
\zeta\left(2^{\alpha} m\right) & =(\alpha+1) \zeta(m) ; \\
N_{0}[n=a x y+b y z+c x] & =N_{1}[n=a x y+b y z+c z x]+\zeta(n / a)+\zeta(n / b)+\zeta(n / c) .
\end{aligned}
$$

In particular

$$
\begin{aligned}
N_{0}[n=x y+y z+2 z x] & =N_{1}[n=x y+y z+2 z x]+2 \zeta(n)+\zeta(n / 2), \\
N_{0}[n=x y+2 y z+2 z x] & =N_{1}[n=x y+2 y z+2 z x]+2 \zeta(n / 2)+\zeta(n) .
\end{aligned}
$$

\footnotetext{
* Presented to the Society, November 29, 1929; received by the editors, September 14, 1929.
} 
3. The numbers $N_{j}(j=0,1)$ have hitherto been determined only in the case $(a, b, c)=(1,1,1)$. As the results for this case will be required we summarize them.*

As always henceforth, $F(n)$ is the number of uneven classes of binary quadratic forms for the determinant $-n$, with all the conventions of $\mathrm{H}$. J. S. Smith's Report on the Theory of Numbers. $\dagger$

$$
\begin{aligned}
& \text { SUMMARY A } \\
& N_{j}(n) \equiv N_{j}[n=x y+y z+z x] \quad(j=0,1) . \\
& N_{j}\left(2^{2 \alpha+1} m\right)=3\left[\left(2^{\alpha+1}-1\right) F(2 m)+(-1)^{j}(\alpha+1) \zeta(m)\right] \text {. } \\
& m \equiv 1 \bmod 4: \quad N_{j}\left(2^{2 \alpha} m\right)=3\left[\left(2^{\alpha+1}-1\right) F(m)+(-1)^{j \frac{1}{2}}(2 \alpha+1) \zeta(m)\right] . \\
& m \equiv 3 \bmod 8: \quad N_{j}\left(2^{2 \alpha} m\right)=3\left[2\left(2^{\alpha}-\frac{1}{3}\right) F(m)+(-1)^{j \frac{1}{2}}(2 \alpha+1) \zeta(m)\right] . \\
& m \equiv 7 \bmod 8: \quad N_{j}\left(2^{2 \alpha} m\right)=3\left[2^{\alpha+1} F(m)+(-1)^{j \frac{1}{2}}(2 \alpha+1) \zeta(m)\right] .
\end{aligned}
$$

These come at once from the formulas XIV quoted, or from Gage's form, on using the following easily seen reduction formulas, in which $G(n)$ is the whole number of classes of binary quadratic forms for the determinant $-\boldsymbol{n}$.

$$
\begin{array}{llrl}
G\left(2^{2 \alpha+1} m\right)=\left(2^{\alpha+1}-1\right) F(2 m) ; & F\left(2^{2 \alpha} m\right)=2^{\alpha} F(m) ; & F\left(2^{2 \alpha+1} m\right)=2^{\alpha} F(2 m) . \\
m & \equiv 1 \bmod 4: & G\left(2^{2 \alpha} m\right) & =\left(2^{\alpha+1}-1\right) F(m) . \\
m & \equiv 3 \bmod 8: & G\left(2^{2 \alpha} m\right) & =2\left(2^{\alpha}-\frac{1}{3}\right) F(m) . \\
m & \equiv 7 \bmod 8: & G\left(2^{2 \alpha} m\right) & =2^{\alpha+1} F(m) .
\end{array}
$$

As implied, the cases $(a, b, c)=(1,1,1),(1,1,2),(1,2,2)$ are intimately connected by the method which we shall indicate. For the last two we find the following.

$$
\begin{aligned}
\text { Summary B } & \\
N_{j}(n) & \equiv N_{j}[n=x y+y z+2 z x] \\
N_{j}(m) & =F(2 m)+(-1)^{i \zeta}(m) . \\
N_{j}\left(2^{2 \alpha+2} m\right) & =\left(2^{\alpha+3}-3\right) F(2 m)+(-1)^{j}(3 \alpha+4) \zeta(m) .
\end{aligned} \quad(j=0,1) .
$$

* See Dickson, History of the Theory of Numbers, vol. 3, p. 224; Gage, American Journal of Mathematics, vol. 51 (1929), pp. 345-348. In the last, make the obvious correction on p. 348 of $\frac{1}{2}(\alpha+1)$ for $\left(\alpha+\frac{1}{2}\right)$. Summary A contains the formulas which I suppressed in my paper, Tôhoku Mathematical Journal, vol. 19 (1921), pp. 105-116, as being (obviously) "rather less simple" than the elegant theorem given there for prime values of $n$. Until after seeing Mordell's paper, American Journal of Mathematics, vol. 45 (1923), pp. 1-4, I did not notice that all cases can be included in a single formula, say that given by Gage. The results follow even more simply than in the last from the formulas XIV of the paper cited in $\$ 6$. There is now no need to reproduce this derivation, as it is superseded by those of Mordell and Gage.

† Mathematical Papers, vol. 1. We have used the elementary reduction formulas on p. 323. See also Dickson's History, vol. 3, chapter 6, where the final notation of Kronecker, used here, is explained. 


$$
\begin{aligned}
& m \equiv 1 \bmod 4: N_{j}\left(2^{2 \alpha+1} m\right)=\left(2^{\alpha+3}-3\right) F(m)+(-1)^{j \frac{1}{2}(6 \alpha+5) \zeta(m) .} \\
& m \equiv 3 \bmod 8: N_{j}\left(2^{2 \alpha+1} m\right)=2\left(2^{\alpha+2}-1\right) F(m)+(-1)^{j \frac{1}{2}}(6 \alpha+5) \zeta(m) . \\
& m \equiv 7 \bmod 8: N_{j}\left(2^{2 \alpha+1} m\right)=2^{\alpha+3} F(m)+(-1)^{j \frac{1}{2}}(6 \alpha+5) \zeta(m) .
\end{aligned}
$$

$$
\begin{aligned}
& N_{j}(n) \equiv N_{j}[n=x y+2 y z+2 z x] \quad(j=0,1) . \\
& N_{j}(m)=\frac{1}{2}\left[2 F(m)+(-1)^{j} \zeta(m)\right] \text {. } \\
& N_{j}(2 m)=2\left[F(m)+(-1)^{i \zeta}(m)\right] \text {. } \\
& N_{j}\left(2^{2 \alpha+3} m\right)=\left[2^{\alpha+2}+3\left(2^{\alpha+1}-1\right)\right] F(2 m)+(-1)^{j}(3 \alpha+5) \zeta(m) . \\
& \left.m \equiv 1 \bmod 4: N_{j}\left(2^{2 \alpha+2} m\right)=\left[2^{\alpha+2}+3\left(2^{\alpha+1}-1\right)\right] F(m)+(-1)^{j \frac{1}{2}(6 \alpha}+7\right) \zeta(m) \text {. } \\
& m \equiv 3 \bmod 8: N_{j}\left(2^{2 \alpha+2} m\right)=2\left[2^{\alpha+1}+3 \cdot 2^{\alpha}-1\right] F(m)+(-1)^{j \frac{1}{2}(6 \alpha+7) \zeta(m)} \text {. } \\
& m \equiv 7 \bmod 8: N_{j}\left(2^{2 \alpha+2} m\right)=5 \cdot 2^{\alpha+1} F(m)+(-1)^{j \frac{1}{2}(6 \alpha+7) \zeta(m)} \text {. }
\end{aligned}
$$

We do not express the foregoing in terms of $G$, as $F$ is the simplest of the class number functions and is that usually tabulated.

4. The results in Summaries B, C follow immediately from Summary A by means of the theorems next stated.

TheOREM 1. $N_{1}\left[2^{\alpha} m=x y+y z+2 z x, y\right.$ odd $]=F\left(2^{\alpha+1} m\right)-\zeta(m)$.

Theorem 2. $N_{1}[m=x y+y z+2 z x]=F(2 m)-\zeta(m)$.

This is the obvious corollary for $\alpha=0$ of Theorem 1 .

Theorem 3. $N_{1}[m=x y+2 y z+2 z x]=\frac{1}{2}[2 F(m)-\zeta(m)]$.

These, with many more which, however, are irrelevant for Summaries B, C, are given by the algebra in $\$ 6$ by which these will be instantaneously proved.

\section{Proofs of Summaries B, C}

5. Assuming for a moment the results stated in Summary A and Theorems $1-3$, all of which, as will be seen in $\S 6$, follow from the same simple analysis, we shall deduce Summaries B, C. As stated in $\$ 2$, footnote, it is unnecessary to give details for Summary A.

Consider $N_{1}(n)$ in Summary C. In $N_{1}\left[2^{\alpha+2} m=x y+2 y z+2 z x\right], x y$ must be even. Hence we may have $x$ even, $y$ odd, or $x$ odd, $y$ even, or $x$ even, $y$ even. Thus the $N_{1}$ in question is equal to

$$
\begin{aligned}
N_{1}\left[2^{\alpha+2} m=2 x y+2 y z+4 z x, y \text { odd }\right]+N_{1}\left[2^{\alpha+2} m\right. & =2 x y+4 y z+2 z x, x \text { odd }] \\
+N_{1}\left[2^{\alpha+2} m\right. & =4 x y+4 y z+4 z x],
\end{aligned}
$$

and therefore is equal to 


$$
2 N_{1}\left[2^{\alpha+1} m=x y+y z+2 z x, y \text { odd }\right]+N_{1}\left[2^{\alpha} m=x y+y z+z x\right] .
$$

Similarly,

$$
N_{1}[2 m=x y+2 y z+2 z x]=2 N_{1}[m=x y+y z+2 z x],
$$

since on the left either but not both of $x, y$ may be even; also

$$
\begin{aligned}
N_{1}\left[2^{\alpha+1} m=x y+x z+2 z x\right]=N_{1}\left[2^{\alpha} m\right. & =x y+y z+z x] \\
& +N_{1}\left[2^{\alpha+1} m=x y+y z+2 z x, y \text { odd }\right] .
\end{aligned}
$$

The rest follows from the above by obvious substitutions and reductions in Summary A and Theorems 1-3.

\section{Proofs of Theorems $1-3$}

6. Let $f(x)$ denote a function which is uniform and finite for all integer values $\geqq 0$ of $x$, and which, for such values of $x$, satisfies $f(x)=f(-x)$. Consider all the solutions $\left(t_{1}, \tau_{1}, d_{2} ; \delta_{2}\right)$, in integers $>0$, where $t_{1}, \tau_{1}$ are odd, $d_{2} \delta_{2}$ is even, and $d_{2}, \delta_{2}$ are of opposite parities, of $m=t_{1} \tau_{1}+d_{2} \delta_{2}$. Then

$$
\begin{aligned}
2 \sum F\left(m-a^{2}\right) f(2 a) & =\zeta(m) f(0)+2 \sum\left[\sum_{r=1}^{(r-1) / 2} f(2 r)\right] \\
& +\sum^{\prime}(\delta-d) f(\delta+d)+\sum^{\prime}\left[f\left(\delta_{2}+d_{2}-\tau_{1}\right)-f\left(\delta_{2}+d_{2}+\tau_{1}\right)\right],
\end{aligned}
$$

in which $\sum$ on the left refers to $a=0, \pm 1, \pm 2, \cdots, \pm\left[m^{1 / 2}\right]$, where $[x]$ is the greatest integer in $x$; $\sum$ on the right refers to all divisors $\tau>0$ of $m$; the first $\sum^{\prime}$ refers to all divisors $d, \delta>0$ of $m$ such that $m=d \delta, d<\delta$, and the second $\sum^{\prime}$ refers to all sets $\left(\delta_{2}, d_{2}, \tau_{1}\right)$.

In this formula take for $f(x)$ the special case $f(0)=1, f(x)=0, x \neq 0$. A straightforward interpretation of the result gives Theorem 3. In precisely the same way Theorem 1 follows from the next.

Consider all the solutions, for $l$ constant, $>0$, and even, of $l=l_{2}+l_{3}$, where $l_{2}, l_{3}$ are $>0$ and even, and (all letters denote integers $>0$ ), $l=t \tau=d \delta$ $=d_{1} \delta_{1} ; d, \delta$ are of like parities, $d<\delta ; \tau$ is odd; $d_{1}, \delta_{1}$ are of opposite parities; $l_{2}=\hat{\iota}_{2} \tau_{2}, \tau_{2}$ is odd $; l_{3}=d_{3} \delta_{3}$, and $d_{3}, \delta_{3}$ are of opposite parities :

Then

$$
l=t_{2} \tau_{2}+d_{3} \delta_{3} \text {. }
$$

$$
\begin{aligned}
4 \sum F(l- & \left.a^{2}\right) f(2 a)=2 \sum\left[f(0)+2 \sum_{r=0}^{(r-1) / 2} f(2 r)\right] \\
& +\sum^{\prime}\left[f(0)+2\left[\sum_{r=1}^{\left(\delta_{1}+d_{1}-1\right) / 2} f(2 r)\right]\right. \\
& +2 \sum^{\prime}(\delta-d) f(\delta+d)+2 \sum^{\prime}\left[f\left(\delta_{3}+d_{3}-\tau_{2}\right)-f\left(\delta_{3}+d_{3}+\tau_{2}\right)\right]
\end{aligned}
$$


the $\sum$ on the left referring to $a=0, \pm 1, \pm 2, \cdots, \pm\left[l^{1 / 2}\right]$.

The stated $f$-theorems are numbers $\mathrm{XII}_{1}, \mathrm{XII}_{2}$ of a former paper on singly infinite class number relations. ${ }^{*}$ As already stated, the formulas XIV of that paper give immediately (inter alia) Summary A.

7. The method mentioned in the introduction is now sufficiently obvious. It may be of interest to indicate a few equally evident consequences of this procedure. First, it is clear that a change of $q$ into $-q$ in an elliptic theta identity from which a paraphrase is inferred, can give no new paraphrases; the arithmetical information contained in both is necessarily the same. Second, to introduce coefficients divisible by a given integer $b$ into the quadratic or bilinear forms relating to a given paraphrase, it is necessary and sufficient to apply to the original theta identity the transformation of order $k b$ ( $k \equiv$ an integer) before paraphrasing or, what is equivalent, to replace $f(x)$ by $\cos x z$ after paraphrasing, and assign to $z$ successively the values $2 \pi / \beta$, where $\beta$ is divisible only by those primes that divide $b$. Third, if instead of $f(x)$, the paraphrase concerns odd functions $g(x)=-g(-x)$, the resulting information, obtained on taking $g(x)=1,0$, or -1 according as $x>0, x=0$ or $x<0$, refers to differences of specialized numbers of representations in the same forms.

The sets of forms simultaneously treated are given by the transformation of any preassigned order.

It is clear that other choices of $f(x)$, as for example $f(x)=1$ (this does not apply in the present case, but it does in all paraphrases obtained from products of even theta quotients exclusively), or $f(x)=x^{2}$, or $x^{4}, \cdots$, give further theorems on numbers of representations. That all are merely aspects of single formulas illustrates the power of the method.

The number of theta expansions in the literature which give simple, interesting arithmetical results is not great. This number has recently been very considerably extended. The works cited put a large number of interesting arithmetical forms within range of exact treatment. $\dagger$

* Quarterly Journal of Mathematics, vol. 49 (1923), pp. 322-337, especially p. 335.

$\dagger$ Three theses: D. A. F. Robinson (Chicago), for the 48 of the 64 functions $\theta_{\alpha}(x+y) /\left[\theta_{\beta}(x) \theta_{\gamma}(x)\right]$ not already expanded by Jacobi, Hermite, Krause and others; J. D. Elder, and M. A. Basoco (California Institute of Technology), who have greatly amplified the work of Biehler, Hermite, Appell, Krause and others on doubly periodic functions of the third kind. Abstracts of the last two are given in the Proceedings of the National Academy of Sciences, vol. 15 (1929), No. 8, pp. 668-671, 675-677.

California Institute of Technology, Pasadena, Calip. 